\title{
Solvatochromism of the Green Fluorescence Protein Chromophore and its Derivatives
}

\author{
Jian Dong, Kyril M. Solntsev, ${ }^{*}$ Laren M. Tolbert* \\ School of Chemistry and Biochemistry, Georgia Institute of Technology, Atlanta, GA 30332-0400 USA.
}

\section{Supporting Information}

\section{General Experimental}

4-Hydroxybenzaldehyde (99\%), p-anisaldehyde (99+ \%), N-acetylglycine (99\%), and methyl trifluoromethanesulfonate (96\%) were purchased from Acros. Sodium acetate $(99.7 \%)$ and potassium carbonate $(99.94 \%)$ were purchased from Fisher. Methylamine (33 \% wt. solution in absolute ethanol) was purchased from Aldrich. The progression of reactions was monitored by thin-layer-chromatography using Analtech UNIPLATE TLC plates (Silica Gel GF) with visualization by illumination with ultraviolet light. NMR spectra were recorded on a Varian Mercury spectrometer $(300 \mathrm{MHz}$ ). Mass spectral analysis was provided by the Georgia Tech Mass Spectrometry facility. Elemental analyses were conducted at Atlantic Microlab. Ultraviolet-visible (UV-Vis) spectra were obtained on a Perkin-Elmer Lambda 19 spectrophotometer.

\section{Synthesis}

4-Methoxybenzylidene-2-methyl-oxazalone. $\quad N$-Acetylglycine $(2.0 \mathrm{~g})$, sodium acetate $(1.23 \mathrm{~g}), \mathrm{p}$-Anisaldehyde $(1.9 \mathrm{~g})$, and acetic anhydride $(4.2 \mathrm{ml})$ were heated at $80{ }^{\circ} \mathrm{C}$ with stirring for $2 \mathrm{~h}$. Heated Ethanol $(20 \mathrm{ml})$ was added slowly and the mixture was allowed to stand at room temperature for $4 \mathrm{~h}$. The separated crystalline solid was filtered off, washed with ice-cold alcohol, then with hot water to obtain $1.4 \mathrm{~g}(44 \%)$ of yellow oxazalone; ${ }^{1} \mathrm{H}$ NMR $\left(\mathrm{CDCl}_{3}\right), \delta$, ppm: 2.40 (s, 3H, $\left.\mathrm{CH}_{3}\right) ; 3.88\left(\mathrm{~s}, 3 \mathrm{H}, \mathrm{O}-\mathrm{CH}_{3}\right) ; 6.98$ (d, 2H, Ar-H); 7.12 (s, 1H, H on bridging carbon); 8.08 (d, 2H, Ar-H).

\section{4-Methoxybenzylidene-1,2-dimethyl-imidazolinone ( $p$-MeOBDI).}

$1.0 \mathrm{~g}$ of 4-(4- Methoxybenzylidene)-2-methyl-oxazalone was added to $0.4 \mathrm{~g}$ of potassium carbonate in a $25 \mathrm{ml}$ round-bottom flash to which $0.75 \mathrm{~g}$ methylamine $(33 \% \mathrm{wt}$. solution in absolute ethanol) and $8.0 \mathrm{ml}$ ethanol were then added. The reaction mixture was refluxed for 4 h. After cooling, the mixture was neutralized with $10 \% \mathrm{HCl}$, and stayed at $0{ }^{\circ} \mathrm{C}$ for a further $4 \mathrm{~h}$. The separated substance was filtered off, washed with ice-cold alcohol, then with hot water to obtain $0.76 \mathrm{~g}(84 \%)$ of dark orange imidazolinone. mp 128-129 ${ }^{\circ} \mathrm{C} .{ }^{1} \mathrm{H}$ NMR $\left(\mathrm{CDCl}_{3}\right), \delta$, ppm: $2.37\left(\mathrm{~s}, 3 \mathrm{H}, \mathrm{CH}_{3}\right) ; 3.18\left(\mathrm{~s}, 3 \mathrm{H}, \mathrm{N}-\mathrm{CH}_{3}\right) ; 3.85$ (s, 3H, O-CH $) ; 6.95$ (d, 2H, Ar-H); 7.08 (s, 1H, $\mathrm{H}$ on bridging carbon); $8.12(\mathrm{~d}, 2 \mathrm{H}, \mathrm{Ar}-\mathrm{H})$. Molecular weight: EI-MS $\mathrm{M}+$ calculated for $\left[\mathrm{C}_{13} \mathrm{H}_{14} \mathrm{~N}_{2} \mathrm{O}_{2}\right]=230.26$, found $=230.1$. 


\section{4-Hydroxybenzylinene-1,2,3-trimethyl-imidazolinium ( $p$-HBDIMe+).}

$200 \mathrm{mg}$ of 4-(4-Hydroxybenzylidene)-1,2-dimethyl-imidazolinone ( $p$-HBDI) was dissolved in $10 \mathrm{ml}$ dry 1,4-dioxane to which 5 drops of methyl trifluoromethanesulfonate were then added. The mixture was stirring at room temperature for $1 \mathrm{~h}$. The separated substance was filtered off, washed with ice-cold alcohol to obtain $185 \mathrm{mg}$ of deep yellow imidazolinium. Decomposed above $175{ }^{\circ} \mathrm{C} .{ }^{1} \mathrm{H}$ NMR spectrum $\left(\mathrm{D}_{2} \mathrm{O}\right), \delta$, ppm: $2.63\left(\mathrm{~s}, 3 \mathrm{H}, \mathrm{CH}_{3}\right) ; 3.28(\mathrm{~s}, 3 \mathrm{H}$, $\left.\mathrm{N}-\mathrm{CH}_{3}\right) ; 3.58\left(\mathrm{~s}, 3 \mathrm{H}, \mathrm{N}-\mathrm{CH}_{3}\right) ; 6.91(\mathrm{~d}, 2 \mathrm{H}, \mathrm{Ar}-\mathrm{H}) ; 7.49$ (s, 1H, H on bridging carbon); 8.14 (d, $2 \mathrm{H}, \mathrm{Ar}-\mathrm{H})$. Molecular weight: EI-MS $\mathrm{M}+$ calculated for $\left[\mathrm{C}_{13} \mathrm{H}_{15} \mathrm{~N}_{2} \mathrm{O}_{2}{ }^{+}\right]=231.27$, found $=$ 231.1 . 
Table S1. Absorption maxima of synthetic GFP chromophores in various solvents.

\begin{tabular}{|c|c|c|c|c|c|c|c|c|c|c|}
\hline \multirow{2}{*}{$\#$} & \multirow{2}{*}{ Solvent $^{\mathrm{a}}$} & \multirow{2}{*}{$\pi^{*}$} & \multirow{2}{*}{$\beta$} & \multirow{2}{*}{$\alpha$} & \multicolumn{3}{|c|}{$p$-HBDI } & \multicolumn{2}{|c|}{ p-MeOBDI } & \multirow{2}{*}{$\begin{array}{c}\boldsymbol{p} \text {-HBDIMe }+ \\
\text { cation }\end{array}$} \\
\hline & & & & & neutral & cation & anion $^{b}$ & neutral $^{\mathrm{c}}$ & Cation & \\
\hline 1 & $\mathrm{Et}_{2} \mathrm{O}$ & 0.24 & 0.47 & 0 & $\begin{array}{r}27696^{\mathrm{d}} \\
361.0\end{array}$ & $\begin{array}{l}24926 \\
401.2\end{array}$ & $\begin{array}{l}22412^{*} \\
446.2^{*}\end{array}$ & $\begin{array}{c}27708 \\
360.9\end{array}$ & $\begin{array}{r}25063 \\
399.0\end{array}$ & \\
\hline 2 & EtOAc & 0.45 & 0.45 & 0 & $\begin{array}{c}27678 \\
361.3\end{array}$ & $\begin{array}{r}25502 \\
392.1\end{array}$ & $\begin{array}{l}22290^{*} \\
448.6^{*}\end{array}$ & $\begin{array}{c}27629 \\
361.9\end{array}$ & $\begin{array}{r}25317 \\
395.0\end{array}$ & \\
\hline 3 & Toluene & 0.49 & 0.11 & 0 & $\begin{array}{r}27649 \\
361.7\end{array}$ & $\begin{array}{c}24992 \\
400.1 \\
\end{array}$ & $\begin{array}{l}22491 \\
444.6\end{array}$ & $\begin{array}{c}27324 \\
366.0\end{array}$ & $\begin{array}{l}24509 \\
408.0\end{array}$ & \\
\hline 4 & Dioxane & 0.49 & 0.37 & 0 & $\begin{array}{c}27603 \\
362.3\end{array}$ & $\begin{array}{r}25170 \\
397.3\end{array}$ & $\begin{array}{r}23109 \\
432.7\end{array}$ & $\begin{array}{c}27454 \\
364.2\end{array}$ & $\begin{array}{r}25125 \\
398.0\end{array}$ & \\
\hline 5 & $\mathrm{EtOH}$ & 0.54 & 0.77 & 0.83 & $\begin{array}{c}27178 \\
367.9\end{array}$ & $\begin{array}{r}25427 \\
393.3\end{array}$ & $\begin{array}{l}22781 \\
439.0\end{array}$ & $\begin{array}{r}27278 \\
366.6\end{array}$ & $\begin{array}{r}25641 \\
390.0\end{array}$ & $\begin{array}{c}24201 \\
413.2\end{array}$ \\
\hline 6 & $\mathrm{THF}$ & 0.55 & 0.55 & 0 & $\begin{array}{r}27397 \\
365.0 \\
\end{array}$ & $\begin{array}{r}25175 \\
397.2 \\
\end{array}$ & $\begin{array}{r}21940^{*} \\
455.8^{*}\end{array}$ & $\begin{array}{r}27503 \\
363.6 \\
\end{array}$ & $\begin{array}{r}25316 \\
395.0 \\
\end{array}$ & $\begin{array}{r}24759 \\
403.9 \\
\end{array}$ \\
\hline 7 & $\mathrm{MeOH}$ & 0.60 & 0.62 & 0.93 & $\begin{array}{c}27232 \\
367.2 \\
\end{array}$ & $\begin{array}{r}25465 \\
392.7 \\
\end{array}$ & $\begin{array}{r}23364 \\
428.0 \\
\end{array}$ & $\begin{array}{c}27398 \\
365.0 \\
\end{array}$ & $\begin{array}{r}25575 \\
391.0 \\
\end{array}$ & $\begin{array}{r}24390 \\
410.0 \\
\end{array}$ \\
\hline 8 & Acetone & 0.62 & 0.48 & 0.08 & $\begin{array}{r}27595 \\
362.4 \\
\end{array}$ & $\begin{array}{r}25627 \\
390.2 \\
\end{array}$ & $\begin{array}{l}21598^{*} \\
463.0^{*}\end{array}$ & $\begin{array}{c}27599 \\
362.3 \\
\end{array}$ & $\begin{array}{r}25381 \\
394.0 \\
\end{array}$ & $\begin{array}{r}25893 \\
386.2 \\
\end{array}$ \\
\hline 9 & $\mathrm{ACN}$ & 0.66 & 0.31 & 0.19 & $\begin{array}{c}27696 \\
361.1 \\
\end{array}$ & $\begin{array}{r}25591 \\
390.8 \\
\end{array}$ & $\begin{array}{l}22130^{*} \\
451.9^{*}\end{array}$ & $\begin{array}{r}27565 \\
362.8 \\
\end{array}$ & $\begin{array}{r}25445 \\
393.0 \\
\end{array}$ & $\begin{array}{c}25044 \\
399.3\end{array}$ \\
\hline 10 & $\mathrm{CH}_{2} \mathrm{Cl}_{2}$ & 0.73 & 0 & 0.3 & $\begin{array}{c}27794 \\
359.8 \\
\end{array}$ & $\begin{array}{c}25306 \\
395.2 \\
\end{array}$ & $\begin{array}{c}21674^{*} \\
461.4^{*}\end{array}$ & $\begin{array}{r}27366 \\
365.4\end{array}$ & $\begin{array}{r}24331 \\
411.0\end{array}$ & $\begin{array}{r}24143 \\
414.2 \\
\end{array}$ \\
\hline 11 & $\mathrm{C}_{2} \mathrm{H}_{4} \mathrm{Cl}_{2}$ & 0.73 & 0 & 0 & $\begin{array}{r}27670 \\
361.4 \\
\end{array}$ & $\begin{array}{c}25296 \\
395.3 \\
\end{array}$ & $\begin{array}{c}22097^{*} \\
452.6^{*}\end{array}$ & $\begin{array}{c}27356 \\
365.6\end{array}$ & $\begin{array}{c}24450 \\
409.0\end{array}$ & $\begin{array}{c}24254 \\
412.3\end{array}$ \\
\hline 12 & TFE & 0.73 & 0 & 1.51 & $\begin{array}{c}27539 \\
363.1\end{array}$ & $\begin{array}{r}25401 \\
393.7\end{array}$ & & $\begin{array}{c}27497 \\
363.7\end{array}$ & $\begin{array}{c}24876 \\
402.0\end{array}$ & \\
\hline 13 & Pyridine & 0.87 & 0.64 & 0 & $\begin{array}{c}26832 \\
372.7\end{array}$ & & $\begin{array}{r}20724 \\
482.5\end{array}$ & $\begin{array}{c}27214 \\
367.5\end{array}$ & & $\begin{array}{r}26205 \\
381.6\end{array}$ \\
\hline 14 & DMF & 0.88 & 0.69 & 0 & $\begin{array}{c}27166 \\
368.1\end{array}$ & $\begin{array}{c}26461 \\
377.9\end{array}$ & $\begin{array}{l}21662 \\
461.6\end{array}$ & $\begin{array}{c}27265 \\
366.8\end{array}$ & & $\begin{array}{r}26532 \\
376.9\end{array}$ \\
\hline 15 & DMSO & 1.00 & 0.76 & 0 & $\begin{array}{r}26997 \\
370.4\end{array}$ & $\begin{array}{r}26080 \\
383.4\end{array}$ & $\begin{array}{l}21214 \\
471.4\end{array}$ & $\begin{array}{r}27141 \\
368.4\end{array}$ & $\begin{array}{c}26178 \\
382.0\end{array}$ & $\begin{array}{r}26274 \\
380.6\end{array}$ \\
\hline 16 & water & 1.09 & 0.4 & 1.17 & $\begin{array}{r}26855 \\
372.4\end{array}$ & $\begin{array}{r}25842 \\
387.0\end{array}$ & $\begin{array}{r}23479 \\
425.9\end{array}$ & $\begin{array}{c}27196 \\
367.7\end{array}$ & $\begin{array}{r}25773 \\
388.0\end{array}$ & $\begin{array}{r}24631 \\
406.0\end{array}$ \\
\hline
\end{tabular}

${ }^{a}$ Solvent parameters $\pi^{*}, \alpha$, and $\beta$ were taken from: Kamlet, M. J.; Abboud, J.-L. M.; Abraham, M. H.; Taft, R. W. J. Org. Chem. 1983, 48, 2877-2887.

${ }^{\mathrm{b}}$ In presence of 18 -crown-6/KOH complex. An asterisk sign refers to the solvents in which an anion could not produced by adding $\mathrm{KOH}$.

${ }^{c}$ The absorption of $p$-MeOBDI had a very little solvent dependence of this peak, and the regression analysis had a poor correlation coefficient. For this column we used the data from Gaussian fit of the $30 \%$ top portion of the absorption spectra. It resulted in much better correlation coefficient (See Table 1), and surprisingly gave the similar solvatochromic parameters.

${ }^{\mathrm{d}}$ Here and below the bottom lines in italic are the experimental data in $\mathrm{nm}\left(\lambda_{\max }\right)$, while the top lines are the data in wavenumbers obtained by $10^{7} / \lambda_{\max }$, and used for further calculations. 

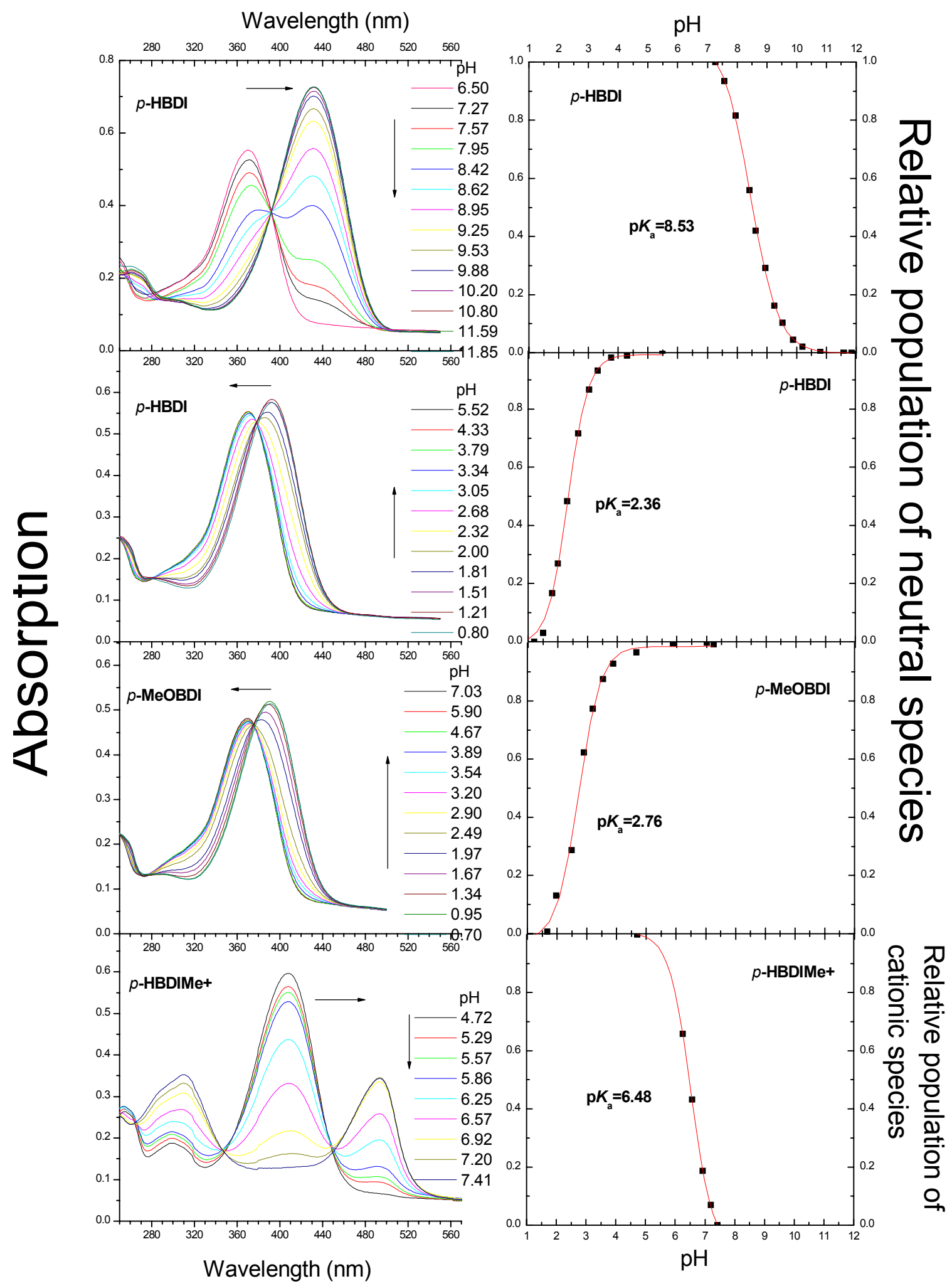

Figure S1. Left column: Absorption spectra of $p$-HBDI and its derivatives at various $\mathrm{pH}$ in $1 / 1$ vol. $\mathrm{MeOH} / \mathrm{H}_{2} \mathrm{O}$ mixtures. Right column: $\mathrm{pH}$-titration curves corresponding to each graph on the left. 


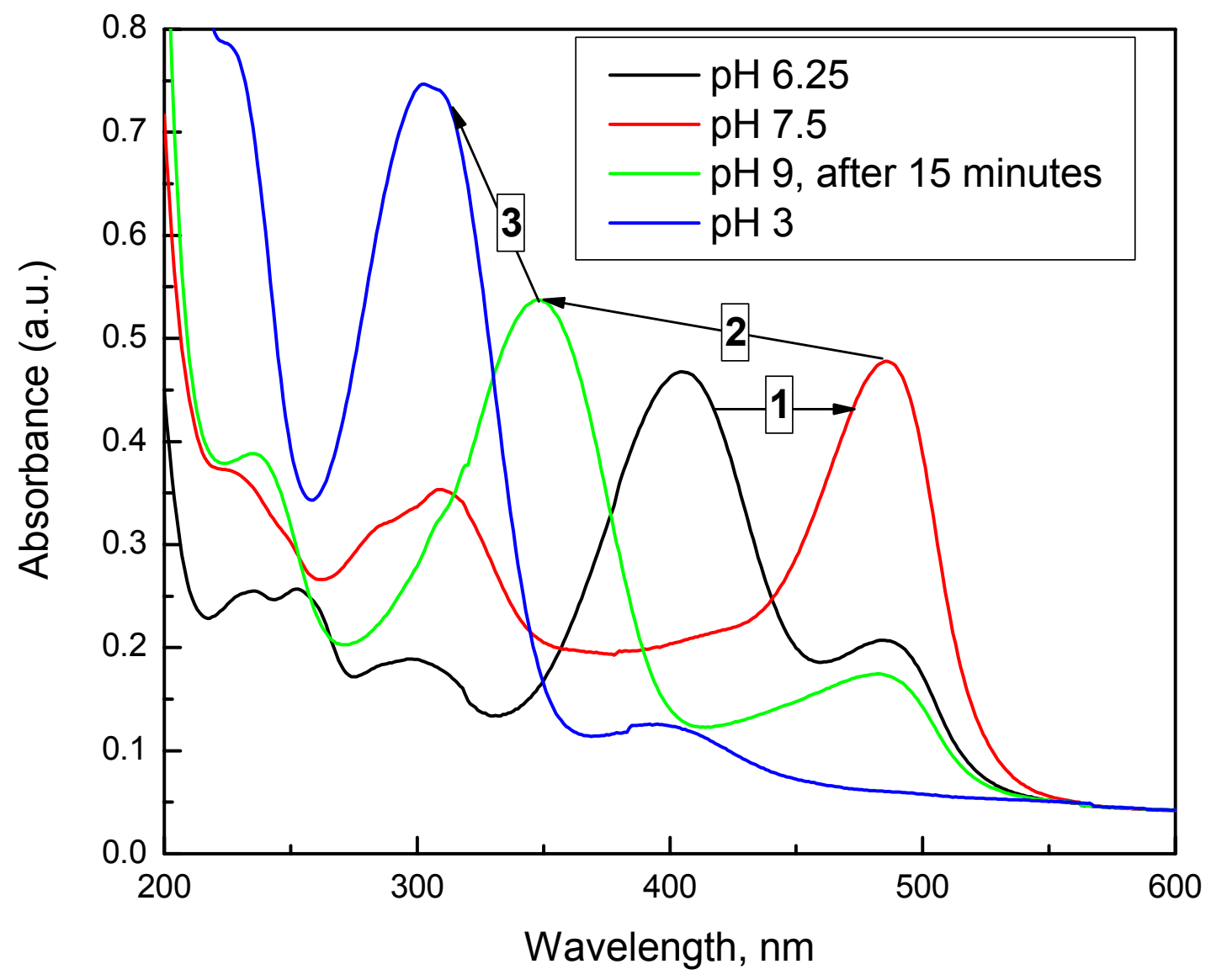

Figure S2. Hydrolysis of $p$-HBDIMe+ in basic conditions. The absorption spectrum of this compound in water at $\mathrm{pH} 6.25$ consisted of absorption bands at $400 \mathrm{~nm}$ and $490 \mathrm{~nm}$, belonging to the cationic $\mathrm{C}$ and zwitterionic $\mathrm{Z}$ form, correspondingly. Step 1: Upon $\mathrm{pH}$ increase to 7.5, the cationic form converted into the zwitterion completely. Step 2: However, upon following $\mathrm{pH}$ increase the intensity of $\mathrm{Z}$ band decreased, and the new absorption band at $350 \mathrm{~nm}$ appeared. Step 3. This band did not belong to parent $p$-HBDIMe + since the initial spectra was not restored after re-acidification to $\mathrm{pH} 3$. Only a weak band at $400 \mathrm{~nm}$ was observed. 


\section{Energy, $1000 / \mathrm{cm}$}

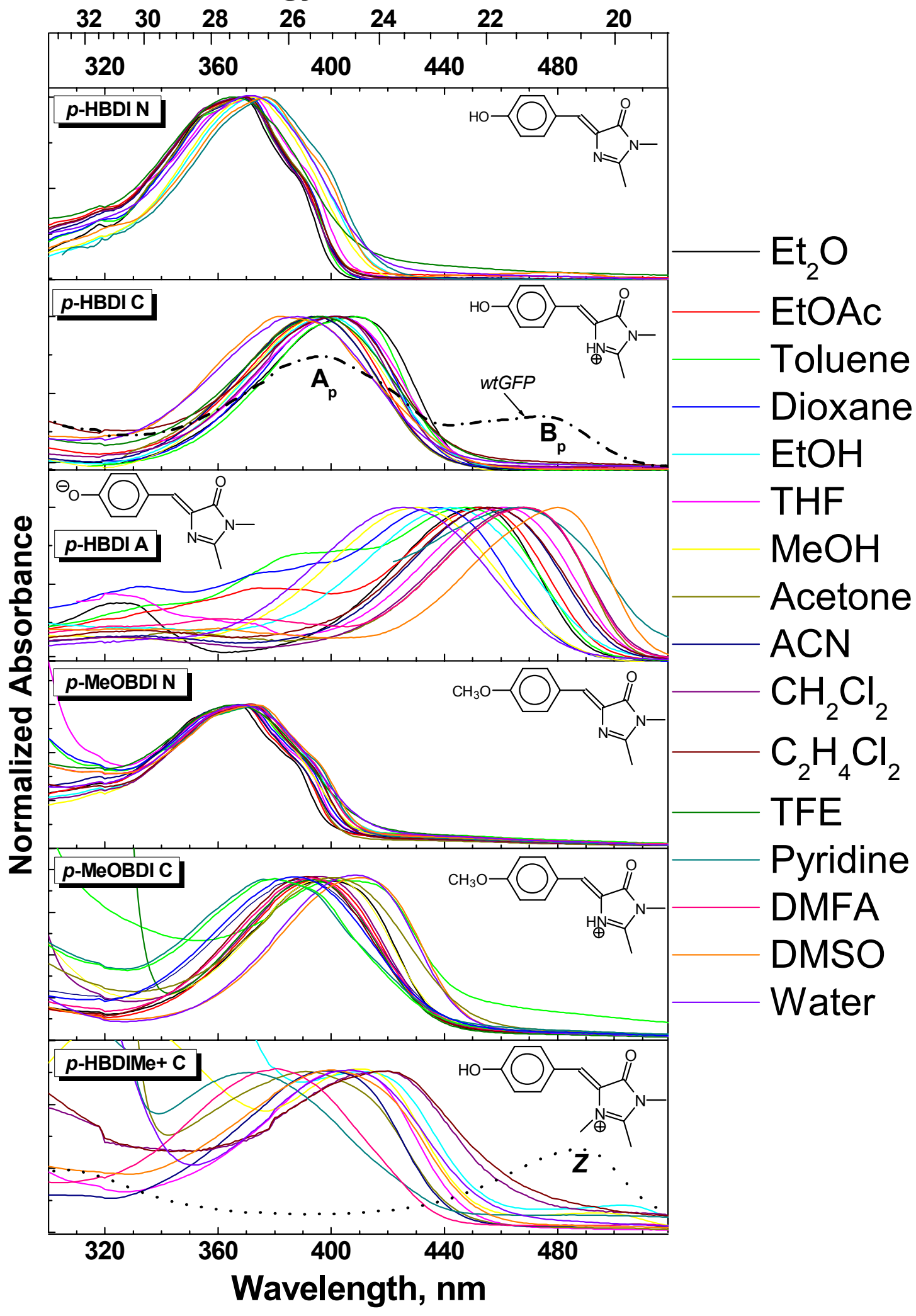

Figure S3. (Expanded version of Figure 1 from the main text). Absorption spectra of different protonation states of $p$-HBDI, $p$-MeOBDI, and $p$-HBDIMe+ in various solvents. For comparison we present the absorption spectra of wt-GFP at $\mathrm{pH} 7$ at "p-HBDI C" graph as a black dash-dotted line. Absorbance of $p$-HBDIMe+ in water at $\mathrm{pH} 7.6$ is presented at the bottom graph as black dotted line, the absorption peak at $486 \mathrm{~nm}$ is due to the zwitterion (Z) absorption. The intensity of the latter is scaled to fit the graph. 

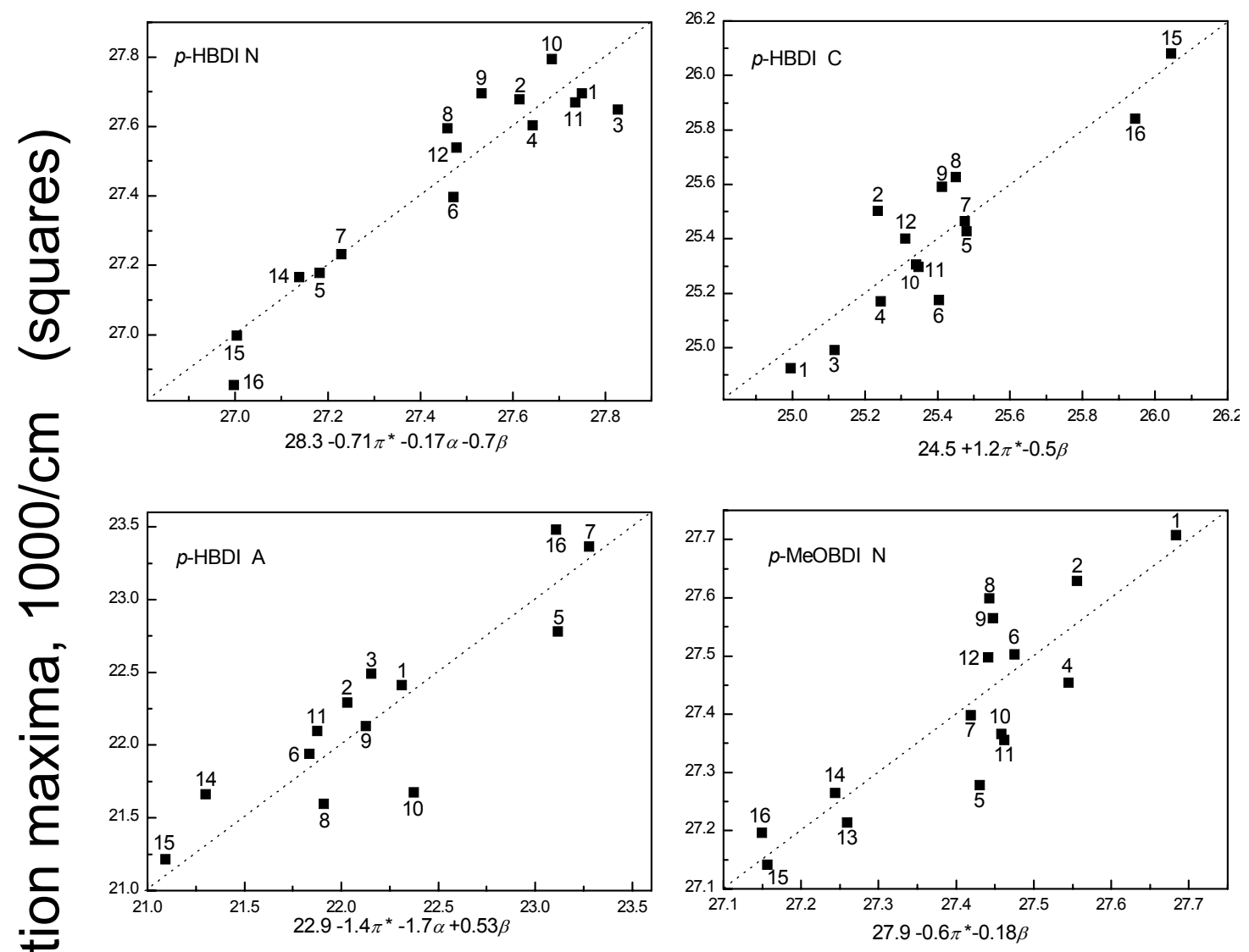

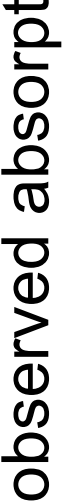
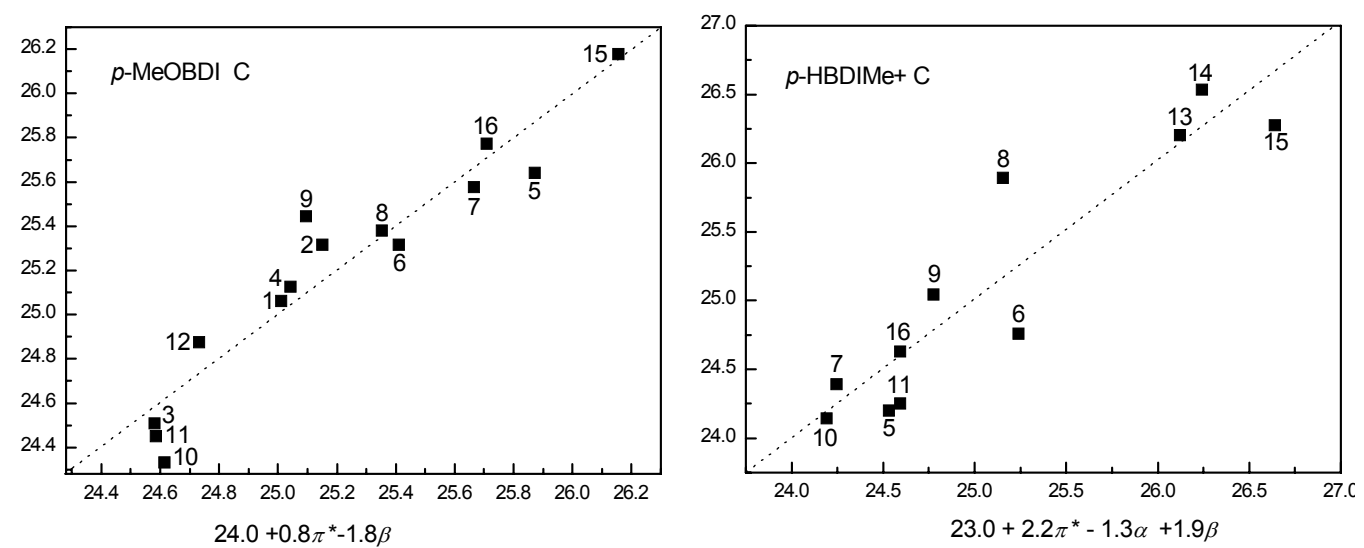

\section{predicted absorption maxima, $1000 / \mathrm{cm}$ (dotted lines)}

Figure S4. Results of multiparameter regression fit according to Eq. 1 (main text). Experimental data (squares) were approximated by the linear combination of $\pi^{*}, \alpha$, and $\beta$ solvents parameters (dotted lines and equations at the bottom of each graph). Each point is labeled corresponding to solvent numbering in Table S1. The correlation coefficients for each fit are listed in Table 1 in the main text. 